\title{
Quasi-Reversibility Regularization Method for Solving a Backward Heat Conduction Problem
}

\author{
Ailin Qian*, Jianfeng Mao \\ School of Mathematics and Statistics, Xianning University, Xianning, China \\ E-mail: junren751113@126.com \\ Received July 15, 2011; revised August 22, 2011; accepted September 2, 2011
}

\begin{abstract}
Non-standard backward heat conduction problem is ill-posed in the sense that the solution (if it exists) does not depend continuously on the data. In this paper, we propose a regularization strategy-quasi-reversibility method to analysis the stability of the problem. Meanwhile, we investigate the roles of regularization parameter in this method. Numerical result shows that our algorithm is effective and stable.
\end{abstract}

Keywords: Back Heat Conduction, Ill-Posed Problem, Quasi-Reversibility, Regularization

\section{Introduction}

In many industrial application one wishes to determine the temperature on the surface of a body, where the surface itself is inaccessible for measurement. The backward heat conduction problem is a model of this situation. In general, no solution which satisfies the heat conduction equation with final data and the boundary conditions exists. Even if a solution exists, it will not be continuously dependent on the final data. The BHCP is a typical example of an ill-posed problem which is unstable by numerical methods and requires special regularization methods. In the context of approximation method for this problem, many approaches have been investigated. Such authors as Lattes and Lions [1], Showalter [2], Ames et al. [3], Miller [4] have approximated the BHCP by quasireversibility methods. Schröter and Tautenhahn [5] established an optimal error estimate for a special BHCP. Mera and Jourhmane used many numerical methods with regularization techniques to approximate the problem in [6-8], etc. A mollification method has been studied by Haö in [9]. Kirkup and Wadsworth used an operatorsplitting method in [10]. So far in the literature, most of the authors used the eigenfunctions and eigenvalues to reconstruct the solution of the BHCP by many quasireversibility methods numerically. However, the eigenfunctions and eigenvalues are in general not available and the labor needed to compute these and the corresponding fourier coefficients is very onerous. In this paper, we use a quasi-reversibility regularization method to solve the BHCP in one-dimensional setting numerically, but this method can be generalized to two-dimensional case.

The paper is organized as follows. In the forthcoming section, we will present the mathematical problem on a BHCP; in Section 2, we review a special quasi-reversibility regularization method; some finite difference schemes are constructed for the inverse problem and the numerical stability analysis is provided; in Section 3, numerical example is tested to verify the effect of the numerical schemes.

\section{Mathematical Problem}

\subsection{The Direct Problem}

We consider the following heat equation:

$$
\begin{array}{ll}
u_{t}(x, t)+u_{x}(x, t)= & u_{x x}(x, t),-\pi<x<\pi, 0<t<T, \\
u(\pi, t)=s(t), & 0<t<T, \\
u(-\pi, t)=l(t), & 0<t<T, \\
u(x, 0)=f(x), & -\pi<x<\pi .
\end{array}
$$

Solving the equation with given $s(t), l(t), f(x)$ is called a direct problem. From the theory of heat equation, we can see that for $s(t), l(t), f(x)$ in some function space there exists a unique solution [11-20].

\subsection{The Inverse Problem}

Consider the following problem:

$$
u_{t}(x, t)+u_{x}(x, t)=u_{x x}(x, t),-\pi<x<\pi, 0<t<T,
$$




$$
\begin{array}{ll}
u(\pi, t)=s(t), & 0<t<T, \\
u(-\pi, t)=l(t), & 0<t<T, \\
u(x, T)=g(x), & -\pi<x<\pi .
\end{array}
$$

The inverse problem is to determine the value of $u(x, t)$ for $0 \leq t<T$ from the data $s(t), l(t), g(x)$. If the solution exists, then the problem has a unique solution [21].

The data $g(x)$ are based on physical observations and are not known with complete accuracy, due to the illposedness of the BHCP, a small error in the data $g(x)$ can cause an arbitrarily large error in the solution $u(x, t)$. Now we want to reconstruct the temperature distribution $u(x, t)$ for $0 \leq t<T$ by quasi-reversibility regularization method.

\section{Quasi-Reversibility Regularization Method}

The initial boundary value problem (2) is replaced by the following problem:

$$
\begin{aligned}
& u_{t}(x, t)+u_{x}(x, t)=u_{x x}(x, t)+\varepsilon u_{x x t}(x, t), \\
& -\pi<x<\pi, 0<t<T, \\
& u(\pi, t)=s(t), \quad 0<t<T, \\
& u(-\pi, t)=l(t), \quad 0<t<T, \\
& u(x, T)=g(x), \quad-\pi<x<\pi .
\end{aligned}
$$

where $\varepsilon$ is a small positive parameter, for $\varepsilon$ sufficiently small the solution of (3) approximates the solution (if it exists) of (2) in some sense. This is one of wellknown quasi-reversibility methods. For the above mentioned problem, Ewing [15] has presented a choice rule of regularization parameter $\varepsilon$, i.e., $\varepsilon=(\ln (1 / \delta))^{-1}$, where $\delta$ denotes the noise level of data $g(x)$, and the error estimate between the approximate solution and the exact solution is given in $L^{2}(R)$-norm.

Take $\tilde{t}=T-t$, then problem (3) becomes

$$
\begin{aligned}
& u_{\tilde{t}}(x, \tilde{t})+u_{x}(x, \tilde{t})=u_{x x}(x, \tilde{t})+\varepsilon u_{x x \tilde{t}}(x, \tilde{t}), \\
& -\pi<x<\pi, 0<\tilde{t}<T, \\
& u(\pi, \tilde{t})=s(\tilde{t}), \quad 0<\tilde{t}<T, \\
& u(-\pi, \tilde{t})=l(\tilde{t}), \quad 0<\tilde{t}<T, \\
& u(x, 0)=g(x), \quad-\pi<x<\pi .
\end{aligned}
$$

The problem has a unique solution if a solution exists. Now we prove it for two-dimensional case.

Theorem 1. There exists a unique solution(if it exists) for the problem:

$$
\begin{aligned}
& u_{\tilde{t}}(x, \tilde{t})-u_{x}(x, \tilde{t})+\Delta u(x, \tilde{t})-\varepsilon \Delta u_{\tilde{t}}(x, \tilde{t})=0, \\
& D \times(0, T),
\end{aligned}
$$

$$
\begin{array}{ll}
u(x, \tilde{t})=h(x, \tilde{t}), & \partial D \times(0, T), \\
u(x, 0)=g(x), &
\end{array}
$$

where $D$ is a bounded subset in $R^{2}, \Delta$ is Laplace operator, $\varepsilon>0$.

Proof. We only need to prove the following problem has the zero solution:

$$
\begin{aligned}
& w_{\tilde{t}}(x, \tilde{t})-w_{x}(x, \tilde{t})+\Delta w(x, \tilde{t})-\varepsilon \Delta u w \tilde{t}(x, \tilde{t})=0, \\
& D \times(0, T), \\
& w(x, \tilde{t})=0, \\
& w(x, 0)=0,
\end{aligned}
$$

Set

$$
\varphi(\tilde{t})=\int_{D} w^{2}(\tilde{t})+\varepsilon|\nabla w(\tilde{t})|^{2} \mathrm{~d} x
$$

then

$$
\frac{\mathrm{d} \varphi(\tilde{t})}{\mathrm{d} \tilde{t}}=2\left(\int_{D} w w_{\tilde{t}}+\varepsilon \nabla w \cdot \nabla w_{\tilde{t}} \mathrm{~d} x\right) .
$$

Due to the Green's second formula, we have

$$
\begin{aligned}
\frac{\mathrm{d} \varphi(\tilde{t})}{\mathrm{d} \tilde{t}} & =2\left(\int_{D} w w_{\tilde{t}}-\varepsilon w \Delta w_{\tilde{t}} \mathrm{~d} x\right) \\
& =2\left(\int_{D} w\left(w_{\tilde{t}}-\varepsilon \Delta w_{\tilde{t}}\right) \mathrm{d} x\right) \\
& =2\left(\int_{D} w\left(w_{x}-\Delta w\right) \mathrm{d} x\right) \\
& =2\left(\int_{D}|\nabla w|^{2} \mathrm{~d} x\right)+2 \int_{D} w w_{x} \mathrm{~d} x \\
& \leq \frac{2}{\varepsilon}\left(\int_{D} w^{2}(\tilde{t})+\varepsilon|\nabla w(\tilde{t})|^{2} \mathrm{~d} x\right) \\
& =\frac{2}{\varepsilon} \varphi(\tilde{t}) .
\end{aligned}
$$

Therefore, we have

$$
\varphi(\tilde{t}) \leq \varphi(0) \mathrm{e}^{\frac{2}{\varepsilon} \tilde{t}} .
$$

since $\varphi(0)=0$, there holds $\varphi(\tilde{t}) \leq 0$, Hence $w=0$.

Now we construct the finite difference schemes for solving problem (4), let $x_{i}=-\pi+(i-1) h, i=1, \cdots 2 n+1$, $\tilde{t}_{j}=(j-1) \tau, j=1, \cdots m+1$, where $h=\pi / n, \tau=T / m$. Let $u_{i}^{j}=u\left(x_{i}, \tilde{t}_{j}\right)$ represent the value of the numerical solution of (4) at the mesh point $\left(x_{i}, \tilde{t}_{j}\right)$, since

$$
\begin{aligned}
& u_{\tilde{t}}\left(x_{i}, \tilde{t}_{j}\right) \simeq \frac{1}{\tau}\left[u\left(x_{i}, \tilde{t}_{j+1}\right)-u\left(x_{i}, \tilde{t}_{j}\right)\right], \\
& u_{x}\left(x_{i}, \tilde{t}_{j}\right) \simeq \frac{1}{h}\left[u\left(x_{i+1}, \tilde{t}_{j}\right)-u\left(x_{i}, \tilde{t}_{j}\right)\right], \\
& u_{x x}\left(x_{i}, \tilde{t}_{j}\right) \simeq \frac{1}{h^{2}}\left[u\left(x_{i-1}, \tilde{t}_{j}\right)-2 u\left(x_{i}, \tilde{t}_{j}\right)+u\left(x_{i}, \tilde{t}_{j}\right)\right],
\end{aligned}
$$




$$
\begin{aligned}
u_{x x \tilde{t}}\left(x_{i}, \tilde{t}_{j}\right) \simeq & \frac{1}{h^{2}}\left\{\frac{1}{\tau}\left[u\left(x_{i-1}, \tilde{t}_{j+1}\right)-u\left(x_{i-1}, \tilde{t}_{j}\right)\right]\right. \\
& -\frac{2}{\tau}\left[u\left(x_{i}, \tilde{t}_{j+1}\right)-u\left(x_{i}, \tilde{t}_{j}\right)\right] \\
& \left.+\frac{1}{\tau}\left[u\left(x_{i+1}, \tilde{t}_{j+1}\right)-u\left(x_{i+1}, \tilde{t}_{j}\right)\right]\right\} .
\end{aligned}
$$

then Equation (4) is discretized as

$$
\begin{aligned}
& -r u_{i+1}^{j+1}+\left(2 r+\frac{1}{\tau}\right) u_{i}^{j+1}-r u_{i-1}^{j+1} \\
= & -\left(\frac{1}{h^{2}}+r+\frac{1}{h}\right) u_{i+1}^{j}+\left(\frac{2}{h^{2}}+2 r+\frac{1}{\tau}+\frac{1}{h}\right) u_{i}^{j} \\
& -\left(\frac{1}{h^{2}}+r\right) u_{i-1}^{j} .
\end{aligned}
$$

where $r=\frac{\varepsilon}{h^{2} \tau}, i=2, \cdots, 2 n, j=1, \cdots, m$.

Now we discuss the stability of difference schemes (8) by verifying the Von Neumann condition. The propagation factor can be found

$$
G(\sigma, \tau)=\frac{\frac{1}{\tau}+4\left(\frac{1}{h^{2}}+r\right) \sin ^{2} \frac{\sigma h}{2}}{\frac{1}{\tau}+4 r_{\sin ^{2}} \frac{\sigma h}{2}} .
$$

It is easy to verify the fact that Von Neumann condition

$$
|G(\sigma, \tau)| \leq 1+c \tau \text {. }
$$

holds with $c=1 / \varepsilon$, hence, the numerical algorithm (8) is stable.

\section{Numerical Examples}

For convenience, we take $s(t)=l(t)=0$ in (2).

Example 1.We consider the following direct problem:

$$
\begin{array}{ll}
u_{t}(x, t)+u_{x}(x, t) & u_{x x}(x, t),-\pi<x<\pi, 0<t<1, \\
u(\pi, t)=0, & 0<t<1, \\
u(\pi, t)=0, & 0<t<1,
\end{array}
$$

with the initial condition:

$$
u(x, 0)= \begin{cases}\pi+x, & -\pi \leq x \leq 0, \\ \pi-x, & 0 \leq x \leq \pi\end{cases}
$$

Let $\quad x_{1}=-\pi, \quad x_{i}=-\pi+(i-1) h, \quad i=1,2, \cdots, 2 n$, $x_{2 n+1}=\pi, \quad t_{j}=(j-1) \tau, \quad j=1,2, \cdots, m+1, \quad$ the space step length $h=\pi / n$, and the time step length $\tau=1 / m$, then we solve this problem by an explicit difference scheme in the following form:

$$
\begin{aligned}
& u_{i}^{j+1}=\frac{(2-h) \tau}{2 h^{2}} u_{i+1}^{j}+\left(1-\frac{2 \tau}{h^{2}}\right) u_{i}^{j}+\frac{(2-h) \tau}{2 h^{2}} u_{i-1}^{j}, \\
& j=1, \cdots, m, \quad i=2, \cdots, 2 n, \\
& u\left(x_{2 n+1, t_{j}}\right)=u_{2 n+1}^{j}=0, \quad 0<t<1, \\
& u\left(x_{1}, t_{j}\right)=u_{1}^{j}=0, \quad 0<t<1,
\end{aligned}
$$

with

$$
u\left(x_{i}, t_{1}=0\right)= \begin{cases}\pi+x_{i}, & -\pi \leq x_{i} \leq 0, \\ \pi-x_{i}, & 0 \leq x_{i} \leq \pi .\end{cases}
$$

where $r=\tau / h^{2}$, and it requires $r<1 / 2$ for numerical stability reasons.

The numerical results for $g(x)=u(x, T=1)$ is shown in Figure 1, where $n=11, m=50$.

Now we solve the inverse problem by the $g(x)$ generated numerical by the direct problem via the quasireversibility regularization method. We choose to restore the solution $f(x)$ at $t=0$. We denotes the numerical results of the inverse problem as $f^{*}(x)$. If we introduce random noises $\varepsilon$ to data $g(x)$, i.e.,

$g_{\varepsilon}\left(x_{i}\right)=g\left(x_{i}\right)+\varepsilon \operatorname{rand}(i)$, where $\operatorname{rand}(i)$ is a random number between $[-1,1]$, then the total noise $\delta$ can be measured in the sense of root mean square error according to:

$$
\delta=\sqrt{\frac{1}{2 n-1} \sum_{i=1}^{2 n-1}\left(g_{\varepsilon}\left(x_{i}\right)-g\left(x_{i}\right)\right)^{2}} .
$$

In the computation, we choose the regularization parameters $h=(\ln (1 / \delta))^{-1}, \delta=0.001$, the numerical results are shown in Figure 2, where $n=11, m=50$.

From Example, we conclude that the choice rules of the regularization parameter $\varepsilon$ is very effective. By our numerical experiment, we can see that the accuracy of the numerical results increases with the decreasing $\mathrm{T}$; at

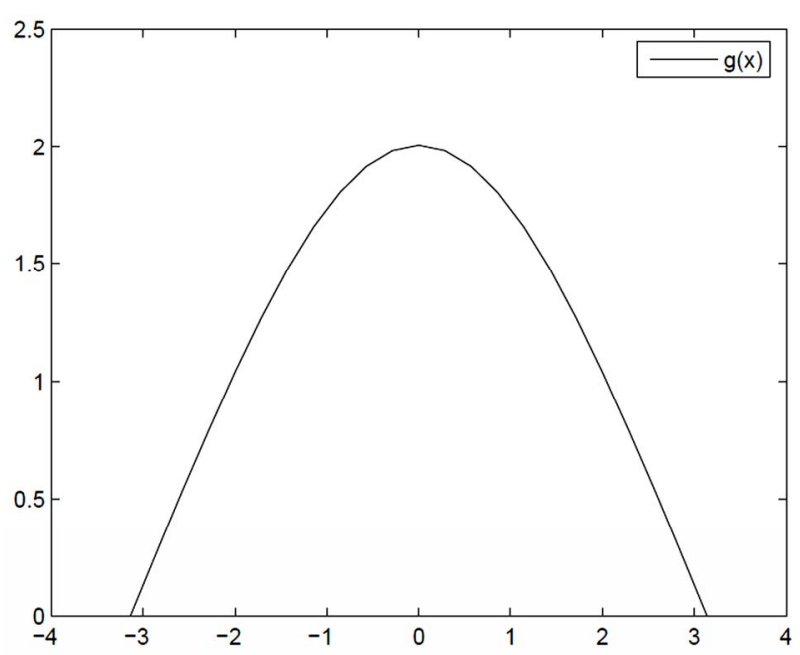

Figure 1. $g(x)$ computed by (13). 


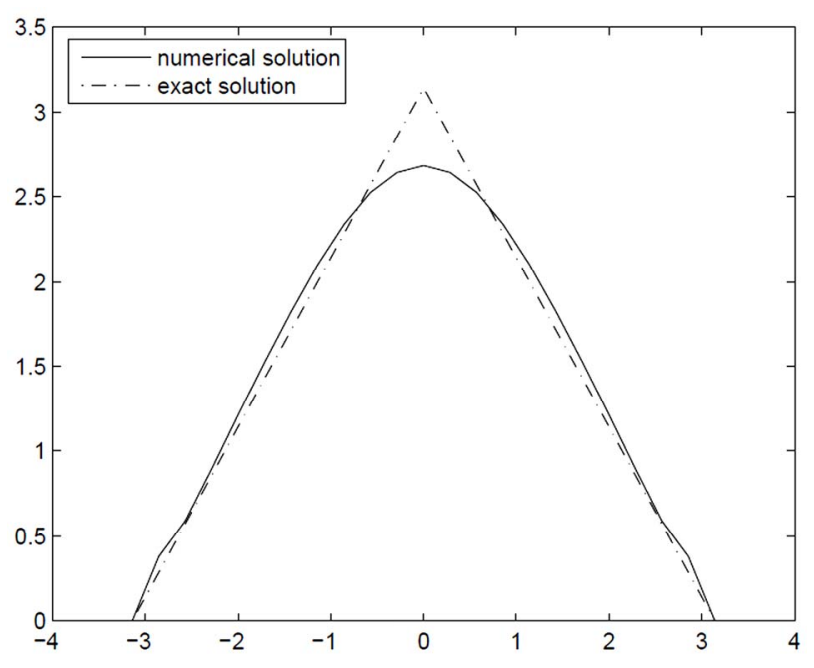

Figure 2. $f^{*}$ and $f, m=50, n=11, \varepsilon=0.145$.

the same time, the numerical solutions of the quasi-reversibility method depend on the parameter $\varepsilon$ continuously. This accords with the theoretical result.

\section{References}

[1] R. Latter and J. L. Lions, "Methode de Quasi-Reversibility et Applications," Dunod, Paris, 1967.

[2] R. E. Showalter, "The Final Value Problem for Evolution Equations," Journal of Mathematical Analysis and Applications, Vol. 47, 1974, pp. 563-572. doi:10.1016/0022-247X(74)90008-0

[3] K. A. Ames, W. C. Gordon, J. F. Epperson and S. F. Oppenhermer, "A Comparison of Regularizations for an Ill-Posed Problem," Mathematics of Computation, Vol. 67, No. 224, 1998, pp. 1451-1471. doi:10.1090/S0025-5718-98-01014-X

[4] K. Miller, "Stabilized Quasireversibility and Other Nearly Best Possible Methods for Non-Well-Posed Problems," Symposium on Non-Well-Posed Problems and Logarithmic Convexity, Lecture Notes in Mathematics, Springer-Verlag, Berlin, Vol. 316, 1973, pp. 161-176.

[5] T. Schröter and U. Tautenhahn, "On Optimal Regularization Methods for the Backward Heat Equation," Zeitschrift für Analysis und ihre Anwendungen, Vol. 15, 1996, pp. 475-493.

[6] N. S. Mera, L. Elliott, D. B. Ingham and D. Lesnic, “An Iterative Boundary Element Method for Solving the One Dimensional Backward Heat Conduction Problem," International Journal of Heat and Mass Transfer, Vol. 44, 2001, pp. 1946-1973.

[7] M. Jourhmane and N. S. Mera, "An Iterative Algorithm for the Backward Heat Conduction Problem Based on Variable Relaxtion Factors," Inverse Problems in Engineering, Vol. 10, No. 4, 2002, pp. 293-308.

\section{doi:10.1080/10682760290004320}

[8] N. S. Mera, "The Method of Fundamental Solutions for the Backward Heat Conduction Problem," Inverse Problems in Engineering, Vol. 13, No. 1, 2005, pp. 79-98. doi:10.1080/10682760410001710141

[9] D. N. Haö, “A Mollification Method for Ill-Posed Problems," Numerische Mathematik, Vol. 68, No. 4, 1994, pp. 469-506. doi:10.1007/s002110050073

[10] S. M. Kirkup and M. Wadsworth, "Solution of Inverse Diffusion Problems by Operator-Splitting Methods," Applied Mathematical Modelling, Vol. 26, No. 10, 2002, pp. 1003-1018. doi:10.1016/S0307-904X(02)00053-7

[11] C. L. Fu, X. T. Xiong and Z. Qian, "Fourier Regularization for a Backward Heat Equation," Journal of Mathematical Analysis and Applications, Vol. 33, No. 1, 2007, pp. 472-481. doi:10.1016/j.jmaa.2006.08.040

[12] L. Elden, "Numerical Solutiono of the Sideways Heat Equation by Diference Approximation in Time," Inverse Problems, Vol. 201, No. 11, 1995, pp. 913-923. doi:10.1088/0266-5611/11/4/017

[13] H. Han, D. B. Ingham and Y. Yuan, "The Boundary Element Method for the Solution of the Backward Heat Conduction Equation," Journal of Computational Physics, Vol. 116, No. 2, 1995, pp. 92-99. doi:10.1006/jeph.1995.1028

[14] M. Jourhmane and N. S. Mera, "An Iterative Algorithm for the Backward Heat Conduction Problem Based on Variable Relaxtion Factors," Inverse Problems in Engneering, Vol. 10, No. 2, 2002, pp. 93-308.

[15] R. E. Ewing, "The Approximation of Cetain Parabolic Equations Backward in Time by Sobolev Equations," SIAM Journal on Mathematical Analysis, Vol. 6, 1975, pp. 283-294. doi:10.1137/0506029

[16] D. N. Hào and P. M. Hien, "Stability Results for the Cauchy Problem for the Laplace Equation in a Strip," Inverse Problems, Vol. 19, No. 4, 2003, pp. 833-844.

[17] D. N. Hào and D. Lesnic, "The Cauchy for Laplace's Equation via the Conjugate Gradient Method," IMA Journal of Applied Mathematics, Vol. 65, 2000, pp. 199. 217. doi:10.1093/imamat/65.2.199

[18] Y. C. Hon and T. Wei, "Backus-Gilbert Algorithm for the Cauchy Problem of Laplace Equation," Inverse Problems, Vol. 17, 2001, pp. 261-271. doi:10.1088/0266-5611/17/2/306

[19] M. V. Klibanov and F. Santosa, "A Computational QuasiReversibility Method for Cauchy Problems for Laplace's Equation," SIAM Journal on Mathematical Analysis, Vol. 51, No. 6, 1991, pp. 1653-1675. doi: $10.1137 / 0151085$

[20] J. R. Cannon, "The One-Dimensional Heat Equation," Addison-Wesley Publishing Company, Menlo Park, 1984.

[21] L. C. Evans, "Partial Differential Equations," American Mathematical Society, Providence, 1998. 